\title{
Screening for transcription factors and their regulatory small molecules involved in regulating the functions of CL1-5 cancer cells under the effects of macrophage-conditioned medium
}

\author{
DONGBO XUE ${ }^{1}, \mathrm{MING} \mathrm{LU}^{2}, \mathrm{BO} \mathrm{GAO}^{1}, \mathrm{XIN} \mathrm{QIAO}^{2}$ and YINGMEI ZHANG ${ }^{3}$ \\ ${ }^{1}$ Department of Surgery, The First Affiliated Hospital of Harbin Medical University, Harbin, \\ Heilongjiang 150001, P.R. China; ${ }^{2}$ Department of Surgery, David Geffen School of Medicine, \\ University of California at Los Angeles, Los Angeles, CA 90095, USA; ${ }^{3}$ Central Laboratory, \\ The First Affiliated Hospital of Harbin Medical University, Harbin, Heilongjiang 150001, P.R. China
}

Received August 15, 2013; Accepted November 26, 2013

DOI: $10.3892 /$ or.2013.2937

\begin{abstract}
Many reports have inferred that macrophages can interact with tumor cells in the tumor microenvironment (TME) in a vicious cycle of tumor development; however, the changes in gene expression in tumor cells under the effects of macrophages are still largely unknown. The present study was carried out to illustrate the changes in the gene expression profile in lung cancer cells under the effects of macrophageconditioned medium. Gene expression profile data were derived from the GEO database GSE9315. The GSM234968 sample was derived from a highly invasive human pulmonary adenocarcinoma cell line, CL1-5, and was treated with conditioned medium (supernatant of a culture solution of human monocyte THP-1). The GSM234967 sample that was not treated with the conditioned medium was used as a control. GO and KEGG enrichment analyses were carried out using DAVID software, and visualization networks were constructed using Cytoscape software. The results showed that 40 differentially expressed genes were annotated. Five differentially expressed transcription factors were identified, EIF2B4, EIF2B5, JUNB, GNG11 and HMGB2, which were all related to 'stress' and 'responses'. The gene cluster of JUNB was mainly enriched in cancer-related pathways, 'Wnt signaling pathway' and 'MAPK signaling pathway'. Finally, 10 small molecules, thioridazine, resveratrol, astemizole, ciclopirox, calmidazolium, etoposide, anisomycin, pyrvinium, azacyclonol and terfenadine, which may act on transcription factors, were identified using the
\end{abstract}

Correspondence to: Professor Yingmei Zhang, Central Laboratory, The First Affiliated Hospital of Harbin Medical University, 23 Youzheng Street, Harbin, Heilongjiang 150001, P.R. China E-mail: zhangyingmei1111@126.com

Key words: macrophage, gene profile, transcription factor, tumor microenvironment
CMap database. In conclusion, we identified transcription factors playing key roles in tumor cells under the effects of macrophages in order to provide new clues for blocking this vicious cycle of tumor development.

\section{Introduction}

Macrophages are important elements in the tumor microenvironment (TME) that are not only affected by cytokines and other cellular products but also produce many of their own cytokines and products to affect survival, proliferation, behavior and the function of tumors and other cells to further affect the regulatory network of cytokines and the TME. It is generally accepted that tumor-associated macrophages (TAM) in the TME are important antitumor effector cells. Mantovani et al (1), proposed the famous 'macrophage balanced hypothesis' in 1992, which suggested that TAMs had dual functions in killing tumors and promoting tumor growth. More and more studies in recent years $(2,3)$ have shown that TAMs are involved in processes associated with the incidence, growth, invasion and migration of tumors, particularly in processes closely related to vascularization and lymph vessel formation in tumors.

Tumor cells can recruit macrophages in the TME, and macrophages can affect tumor cells in return, thus forming a vicious cycle. Measures can be taken to prevent the recruitment of macrophages, attenuate the secretory functions of macrophages, antagonize the cytokines secreted by macrophages, block signaling pathways in tumor cells, in order to block this vicious cycle. However, most of the previous studies have focused on the effects of tumor cells on TAMs and the changes in the cytokines secreted by TAMs. The changes in gene expression in tumor cells under the effects of macrophages are still largely unknown. Therefore, the present study was carried out to illustrate the changes in the gene expression profile in lung cancer cells under the effects of a macrophage-conditioned medium based on microarray data from the Gene Expression Omnibus (GEO) database and to 
find the transcription factors playing key roles to provide new clues for blocking this vicious cycle.

\section{Materials and methods}

Microarray data. The gene expression profile data were derived from the GEO database GSE9315 of the National Center for Biotechnology Information (NCBI) (http://www.ncbi.nlm.nih.gov/geo/query/acc.cgi?acc=GSE9315) submitted by Lee CY et al. Human monocyte THP-1 and a highly invasive human pulmonary adenocarcinoma cell line, CL1-5, were utilized as the subjects for investigation. THP-1 cells were pre-treated with phorbol myristate acetate (PMA) for $24 \mathrm{~h}$ to allow for differentiation into macrophages, and the cells were further incubated in serum-free culture media for another $24 \mathrm{~h}$. After the PMA was washed out, the supernatant of the culture solution was used as the conditioned medium. The GSM234967 sample was derived from CL1-5 cells that were not treated with the conditioned medium, and the sample GSM234968 was derived from CL1-5 cells that were treated with the conditioned medium.

The microarray utilized in the present study was GPL5968, which is a human cDNA CHIP that contains 1,152 human EST clones with putative gene names related to cell adhesion, motility, angiogenesis, signal transduction, tumorigenesis and metastasis.

Methods for microarray data analysis. The microarray analysis was carried out for GSM234967 and GSM234968 samples. The CEL data compression package for microarray was downloaded in the supplementary file of GEO and was decompressed into another folder for further use. Additionally, the original data for the sample were also downloaded in TXT format. The downloaded data were standardized using Bioconductor (version 2.10.1) (3). The RMA algorithm was used to calculate the expression level, and the MAS algorithm was used to calculate the detection call. Samples with no less than 2 detection calls were retained as the $\mathrm{P}$ filter low expression probe number , and human HGNC gene abbreviations were used to unify the gene names. Afterwards, the VLOOKUP function was used as a substitute for the probe number in the original data, and the expression datasheet correlating the gene names and samples was established. The LIMMA differential gene screening algorithm was used to screen for the upregulated genes and the downregulated genes in GSM234968 as compared to GSM234967. The fold-change, P-value and FDR values were calculated.

Bioinformatic analysis of the differentially expressed genes. DAVID software was used for Gene Ontology (GO) and Kyoto Encyclopedia of Genes and Genomes (KEGG) enrichment analyses. The DAVID database (http://david.abcc.ncifcrf.gov/) was opened and the gene cluster was submitted for further analysis. The corresponding gene designator was selected, and the whole genome of the human served as the background. Then, the 'Functional Annotation Tool' was used to obtain the results for the GO and KEGG enrichment analysis (P-value was set to 0.05 ).

The protein-protein interaction (PPI) network was obtained from the HPRD database (http://www.hprd.org/), which contained 36,874 lines and 9,453 nodes. Forty differentially expressed genes were projected into the PPI network, and the correlation pairs that correlated with every differentially expressed gene were retained; thus, a network composed of the nodes was directly connected with the differentially expressed genes.

The GO terms or KEGG pathways of interest and their corresponding genes were selected, and the genes with the same functions were correlated by constructing a visualization network using Cytoscape software (version 2.6.3).

Screening of the regulatory small molecules. The connectivity map (CMap) database consists of data from whole-genome transcription expression profiles of human cells under the influence of active small molecules, including 7,256 expression profiles from 6,100 groups of small molecule interference experiments (a small molecule interference group and the normal control group). In total, 1,309 small molecules are included in the 6,100 groups of small molecule interference experiments

According to the interference experiments on cancer cells using the 1,309 small molecules documented in the CMap database, we analyzed the differentially expressed genes from the expression profile data derived from the pulmonary adenocarcinoma samples (GSM234968 vs. GSM234967). The drugs and the gene sets affected by the drugs (the differentially expressed genes) were obtained. Then, the differences in gene expression for pulmonary adenocarcinoma were analyzed and compared with the differentially expressed genes induced by the interference of the molecules to identify the small molecules that may be driving variations in gene expression between normal and diseased cells.

The genes showing differential expression in the diseased cells in comparison to the normal cells were categorized into downregulated genes and upregulated genes, and then these genes were used to make a probe set under the HG-U133A platform. This probe set was used to compare the differentially expressed genes after treatment with the small molecules in the CMap database, and finally an enrichment value representing similarity was obtained.

\section{Results}

Results of the analysis of the differentially expressed genes. After the analysis of the microarray data, 1,041 genes were analyzed, among which 70 genes showed either an expression increase of at least 2-fold or an expression decrease of at least $50 \%$. The 70 differentially expressed genes were uploaded for functional annotation, and 40 genes were annotated (as shown in Table I): ANXA4, CD1D, CDK2, COMT, GJA1, GNG11, HMGB2, IL12A, IL18, JUNB, STMN1, MMP7, MYBL2, NUBP1, NFKB2, PLAU, RBBP4, RGS4, SMN2, STC1, YWHAH, EIF2B4, EIF2B5, NFS1, AURKB, PTTG1, CIAO1, MRC2, TUBB4B, NID2, RNF167, RPS27L, DPH5, SNIP1, COL21A1, PLA2G12A, RNF135, C1orf87, PCNA-AS1 and SEPT5-GP1BB.

Results of the enrichment analysis of the differentially expressed genes. The 40 genes listed above were subjected to functional enrichment analysis, and the top $15 \mathrm{GO}$ terms 
Table I. Differentially expressed genes in the CL1-5 cells following treatment with the macrophage-conditioned medium.

\begin{tabular}{|c|c|c|c|c|}
\hline & Gene symbol & Entrez ID & Description & Fold-change value \\
\hline 1 & ANXA4 & 307 & Annexin A4 & 2.024670261 \\
\hline 2 & CD1D & 912 & CD1d molecule & 2.309465826 \\
\hline 3 & CDK2 & 1017 & Cyclin-dependent kinase 2 & 2.464015819 \\
\hline 4 & COMT & 1312 & Catechol-O-methyltransferase & 2.967239056 \\
\hline 5 & GJA1 & 2697 & Gap junction protein, $\alpha 1$ & 2.284841855 \\
\hline 6 & GNG11 & 2791 & Guanine nucleotide binding protein (G protein), $\gamma 11$ & 2.593119266 \\
\hline 7 & HMGB2 & 3148 & High mobility group box 2 & 2.421030043 \\
\hline 8 & IL12A & 3592 & $\begin{array}{l}\text { Interleukin } 12 \mathrm{~A} \text { (natural killer cell stimulatory factor } 1 \text {, } \\
\text { cytotoxic lymphocyte maturation factor } 1, \mathrm{p} 35 \text { ) }\end{array}$ & 2.266825069 \\
\hline 9 & IL18 & 3606 & Interleukin 18 (interferon- $\gamma$-inducing factor) & 2.115577065 \\
\hline 10 & JUNB & 3726 & Jun B proto-oncogene & 0.497792634 \\
\hline 11 & STMN1 & 3925 & Stathmin 1 & 2.409299142 \\
\hline 12 & MMP7 & 4316 & Matrix metallopeptidase 7 (matrilysin, uterine) & 2.166427269 \\
\hline 13 & MYBL2 & 4605 & V-myb myeloblastosis viral oncogene homolog (avian)-like 2 & 2.181687983 \\
\hline 14 & NUBP1 & 4682 & Nucleotide binding protein 1 & 2.162368172 \\
\hline 15 & NFKB2 & 4791 & $\begin{array}{l}\text { Nuclear factor of } \kappa \text { light polypeptide } \\
\text { gene enhancer in B-cells } 2(\mathrm{p} 49 / \mathrm{p} 100)\end{array}$ & 0.450935487 \\
\hline 16 & PLAU & 5328 & Plasminogen activator, urokinase & 0.33083469 \\
\hline 17 & RBBP4 & 5928 & Retinoblastoma binding protein 4 & 2.300745137 \\
\hline 18 & RGS4 & 5999 & Regulator of G-protein signaling 4 & 2.030545158 \\
\hline 19 & SMN2 & 6607 & Survival of motor neuron 2 , centromeric & 2.05350515 \\
\hline 20 & STC1 & 6781 & Stanniocalcin 1 & 0.308078625 \\
\hline 21 & YWHAH & 7533 & $\begin{array}{l}\text { Tyrosine } 3 \text {-monooxygenase/tryptophan } 5 \text {-monooxygenase } \\
\text { activation protein, } \eta \text { polypeptide }\end{array}$ & 2.018610231 \\
\hline 22 & EIF2B4 & 8890 & Eukaryotic translation initiation factor $2 \mathrm{~B}$, subunit $4 \delta, 67 \mathrm{kDa}$ & 2.024384748 \\
\hline 23 & EIF2B5 & 8893 & Eukaryotic translation initiation factor $2 \mathrm{~B}$, subunit $5 \varepsilon, 82 \mathrm{kDa}$ & 2.051791918 \\
\hline 24 & NFS1 & 9054 & NFS1 nitrogen fixation 1 homolog (S.cerevisiae) & 2.078554339 \\
\hline 25 & AURKB & 9212 & Aurora kinase B & 2.143919742 \\
\hline 26 & PTTG1 & 9232 & Pituitary tumor-transforming 1 & 2.323462232 \\
\hline 27 & CIAO1 & 9391 & Cytosolic iron-sulfur protein assembly 1 & 0.462791283 \\
\hline 28 & $\mathrm{MRC} 2$ & 9902 & Mannose receptor, $\mathrm{C}$ type 2 & 2.106472697 \\
\hline 29 & TUBB4B & 10383 & Tubulin, $\beta$ 4B class IVb & 2.09408162 \\
\hline 30 & NID2 & 22795 & Nidogen 2 & 0.379487576 \\
\hline 31 & RNF167 & 26001 & Ring finger protein 167 & 2.075793535 \\
\hline 32 & RPS27L & 51065 & Ribosomal protein S27-like & 2.319500882 \\
\hline 33 & DPH5 & 51611 & DPH5 homolog (S. cerevisiae) & 2.095665817 \\
\hline 34 & SNIP1 & 79753 & Smad nuclear interacting protein 1 & 2.314891847 \\
\hline 35 & COL21A1 & 81578 & Collagen, type XXI, $\alpha 1$ & 2.036490338 \\
\hline 36 & PLA2G12A & 81579 & Phospholipase A2, group XIIA & 2.430047715 \\
\hline 37 & RNF135 & 84282 & Ring finger protein 135 & 2.033762661 \\
\hline 38 & C1orf87 & 127795 & Chromosome 1 open reading frame 87 & 0.367687812 \\
\hline 39 & PCNA-AS1 & 100302739 & PCNA antisense RNA 1 & 3.150155333 \\
\hline 40 & SEPT5-GP1BB & 100526833 & SEPT5-GP1BB read through & 3.570031192 \\
\hline
\end{tabular}

were selected (Fig. 1). Nine of these genes were categorized as 'response to abiotic stimulus' (GO: 0009628), 'response to organic substance' (GO: 0010033), 'negative regulation of translational initiation in response to stress' (GO: 0032057), 'response to hormone stimulus' (GO: 0009725), 'negative regulation of translation in response to stress' (GO: 0032055), 'regulation of translational initiation in response to stress' (GO: 0043558), 'response to endogenous stimulus' (GO: 0009719), 'response to temperature stimulus' (GO: 0009266), 'regulation of translation in response to stress' (GO: 0043555), or were correlated with 'stress' and 'responses'. Among these 40 genes, 6 genes (RBBP4, IL12A, AURKB, PTTG1, STMN1 and CDK2) were related to the 'cell cycle' (GO: 0007049, GO: 0051726, GO: 0000279, GO: 0022402, 


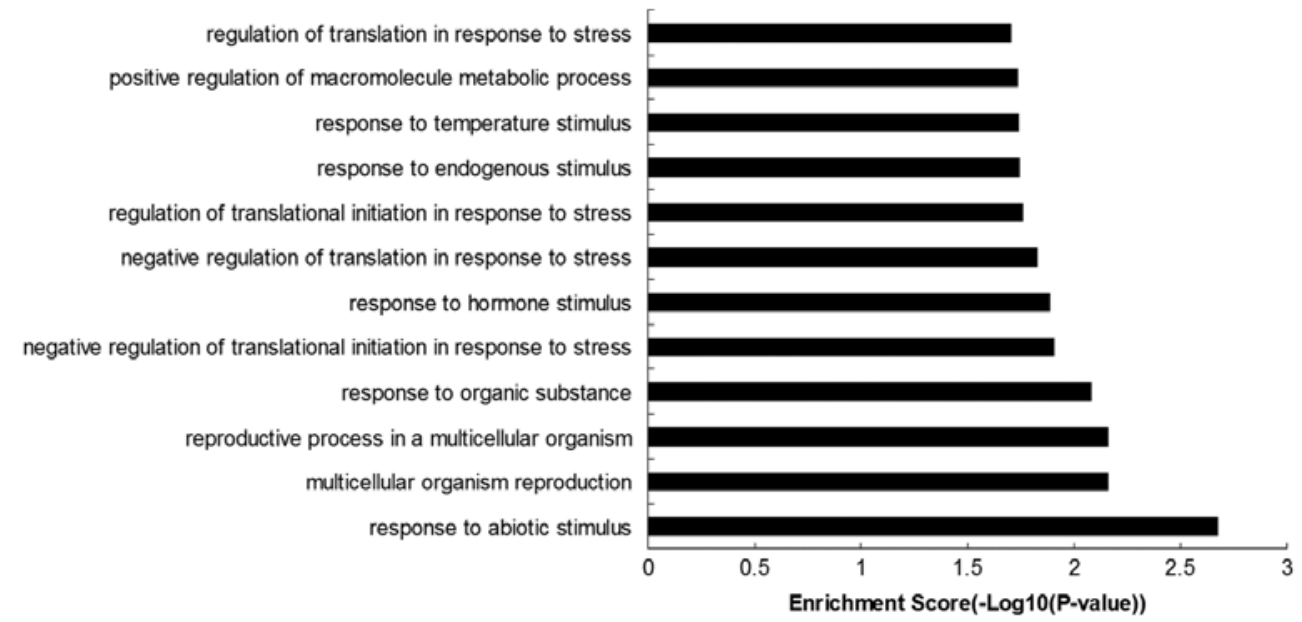

Figure 1. GO biological process enrichment analysis of the 40 differentially expressed genes in CL1-5 cells following treatment with macrophage-conditioned medium. GO, gene ontology.

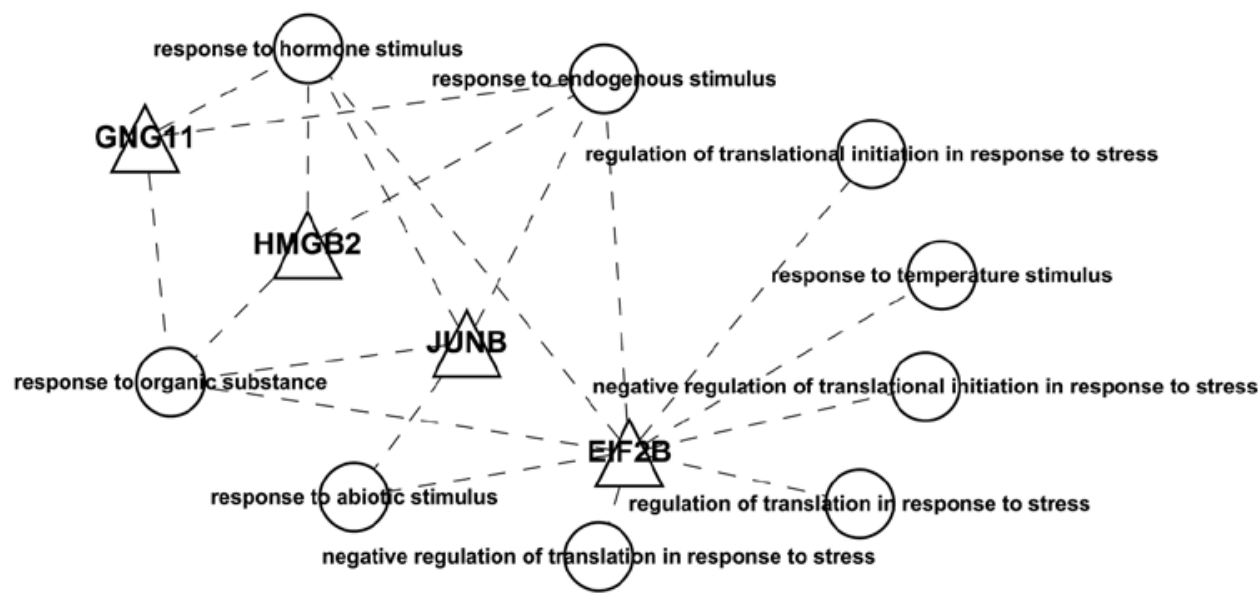

Figure 2. Network for the differentially expressed transcription factors and their GO biological process functions. The ellipse-shaped nodes indicate the GO terms for biological process; the triangle-shaped nodes indicate differentially expressed transcription factors. GO, gene ontology.

GO: 0000278, GO: 0022403); 4 genes (RBBP4, IL12A, GJA1 and COMT) were related to 'cell proliferation' (GO: 0008285); 3 genes (HMGB2, RBBP4 and CDK2) were related to 'DNA replication' (GO: 0006260); 7 genes (HMGB2, YWHAH, RGS4, SP1NI, RPS27L, GNG11 and STMN1) were related to an 'intracellular signaling cascade' (GO: 0007242) and 3 genes (IL18, JUNB and PLAU) were related to 'blood vessel morphogenesis' (GO: 0048514).

An in-depth literature search revealed that TUBB4B, STMN1 and NUBP1 were related to 'the functions of microfilaments and microtubules'; GJA1, MMP7, PLAU, NID2, MRC2 and COL21A1 were related to 'extracellular matrix'; SNIP1, MYBL2, PCNA-AS1, STC1 and COMT were related to 'lung cancer'; RGS4 and GNG11 were related to 'breast cancer'; and GNG11 was related to 'endometrial carcinoma'.

Notably, we found 5 differentially expressed transcription factors: EIF2B4, EIF2B5, JUNB, GNG11 and HMGB2. Using these transcription factors and the GO functional annotations, we constructed a visualization graph (Fig. 2) and found that these transcription factors were all related to 'stress' and 'responses'.
Functional enrichment analysis and the construction of the visualization network for transcription factors and regulatory genes. To better illustrate the functions of the differentially expressed genes and their interactions, we constructed a network composed of the nodes directly connecting the differentially expressed genes (Fig. 3); we found that the differentially expressed genes had no direct interaction, but an interaction network may be constructed by using the expanded nodes. EIF2B, JUNB, GNG11 and HMGB2 and their interacting genes were selected, and the visualization graph is shown in Fig. 4. The KEGG functional enrichment analysis for each gene cluster did not show enrichment for GNG11 and HMGB2, but it did show enrichment for JUNB and EIF2B, as shown in Figs. 5 and 6. The functions of the JUNB transcription factor were mainly enriched for the 'colorectal cancer pathway', 'pathways in cancer', 'pancreatic cancer pathway', 'Toll-like receptor signaling pathway', 'Wnt signaling pathway', 'MAPK signaling pathway', 'NOD-like receptor signaling pathway', 'RIG-I-like receptor signaling pathway', 'Fc epsilon RI signaling pathway', 'GnRH signaling pathway', 'T cell receptor signaling pathway' and 'neurotrophin 

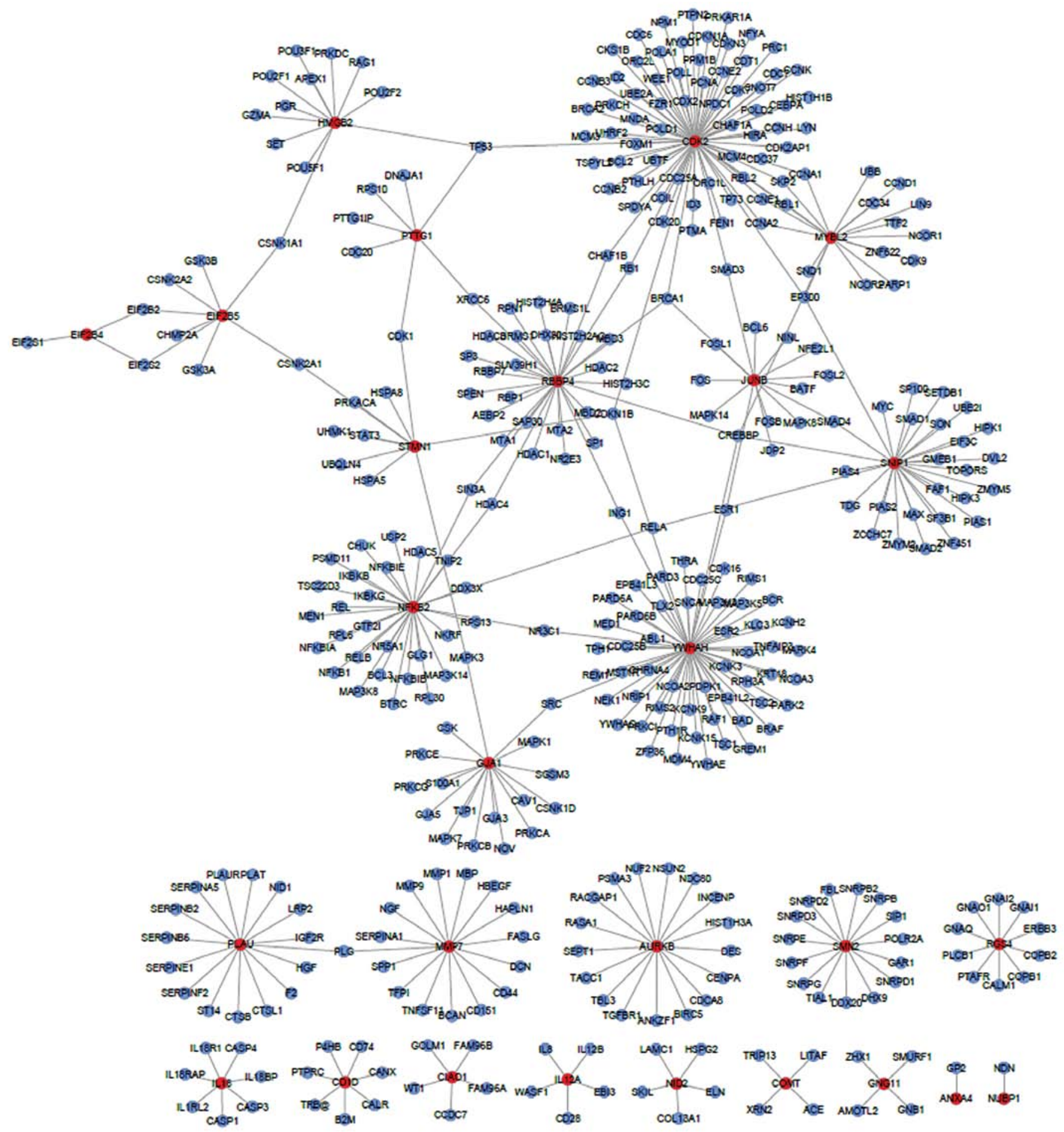

Figure 3. Network of genes that directly links the 40 differentially expressed genes.

signaling pathway'. The functions of the EIF2B-related gene cluster were mainly enriched in 'Wnt signaling pathway' and 'Hedgehog signaling pathway'.

Results for the screening for small molecules regulating transcription factors. Using the 40 differentially expressed genes, a comparison (map) was generated for the genes and the differentially expressed genes interacting with the small molecules and the correlations between each gene and the small molecules were obtained (Fig. 7). The correlations between the transcription factors and the small molecule are shown in Fig. 8.

After the upregulated and downregulated genes for the disease were used to make the probe set under the HG-U133A platform, the CMap database was used to search for diseaserelated small molecules. We used the small molecules showing the highest correlation between small molecule treatments and transcription factors (the P-value is the smallest) as the examples. These small molecules are listed in Fig. 9. Thioridazine, resveratrol, astemizole, ciclopirox, 
A
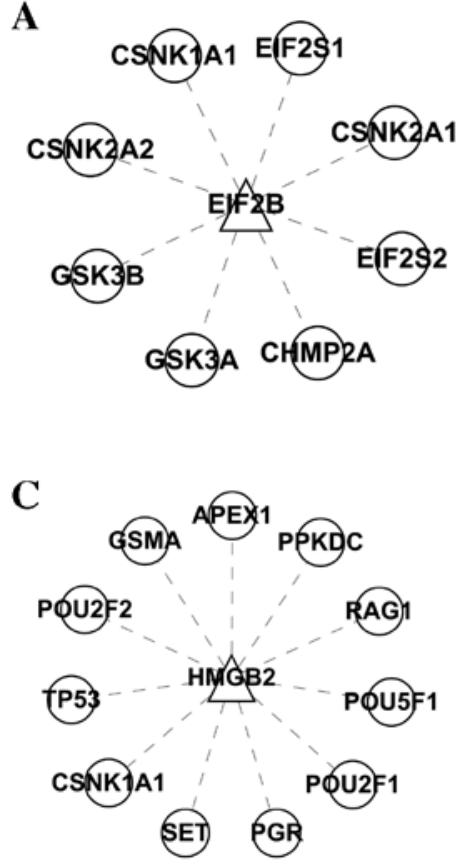

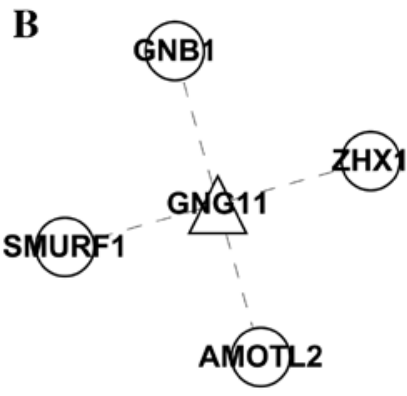

D

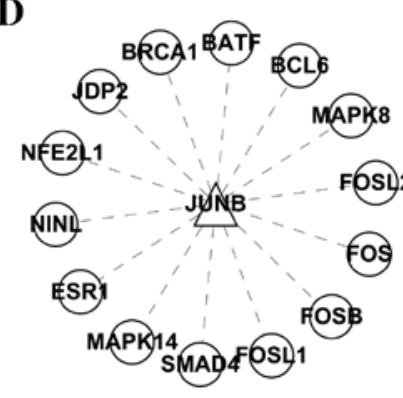

Figure 4. Network of selected transcription factors and their regulatory genes. The ellipse-shaped nodes indicate the regulatory genes of the corresponding transcription factor; the triangle-shaped nodes indicate the differentially expressed transcription factors. The lines "--' indicate functional interactions. (A) EIF2B, (B) GNG11, (C) HMGB2 and (D) JUNB.

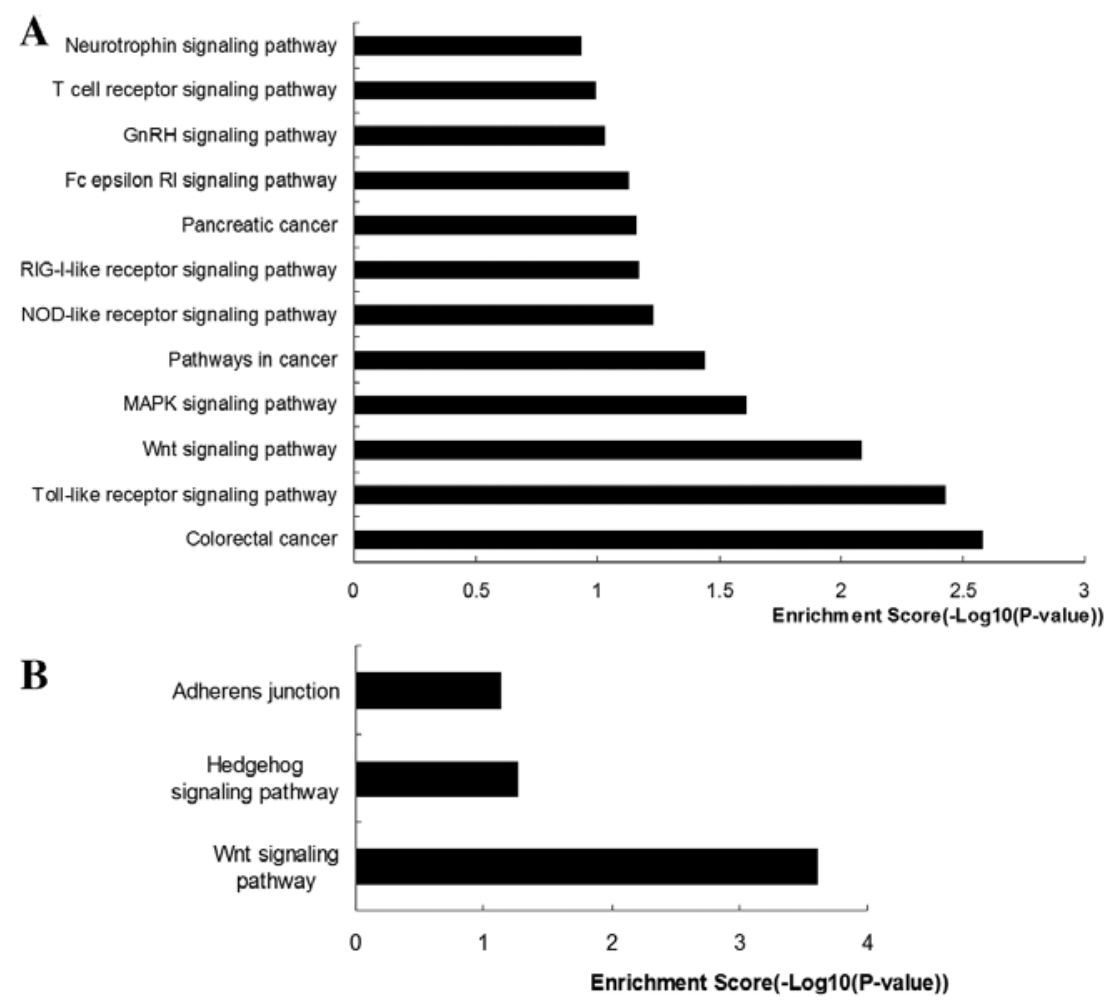

Figure 5. KEGG enrichment analysis of the transcription factors and their regulatory genes. (A) The gene cluster of JUNB; (B) the regulatory gene cluster of EIF2B. KEGG, Kyoto Encyclopedia of Genes and Genomes.

calmidazolium, etoposide, anisomycin, pyrvinium, azacyclonol and terfenadine were negatively correlated, which indicates that these small molecules may be useful for the treatment of disease.

\section{Discussion}

The TME is composed of a series of resident cells, such as fibroblasts and migrating cells, such as hematopoietic cells. 

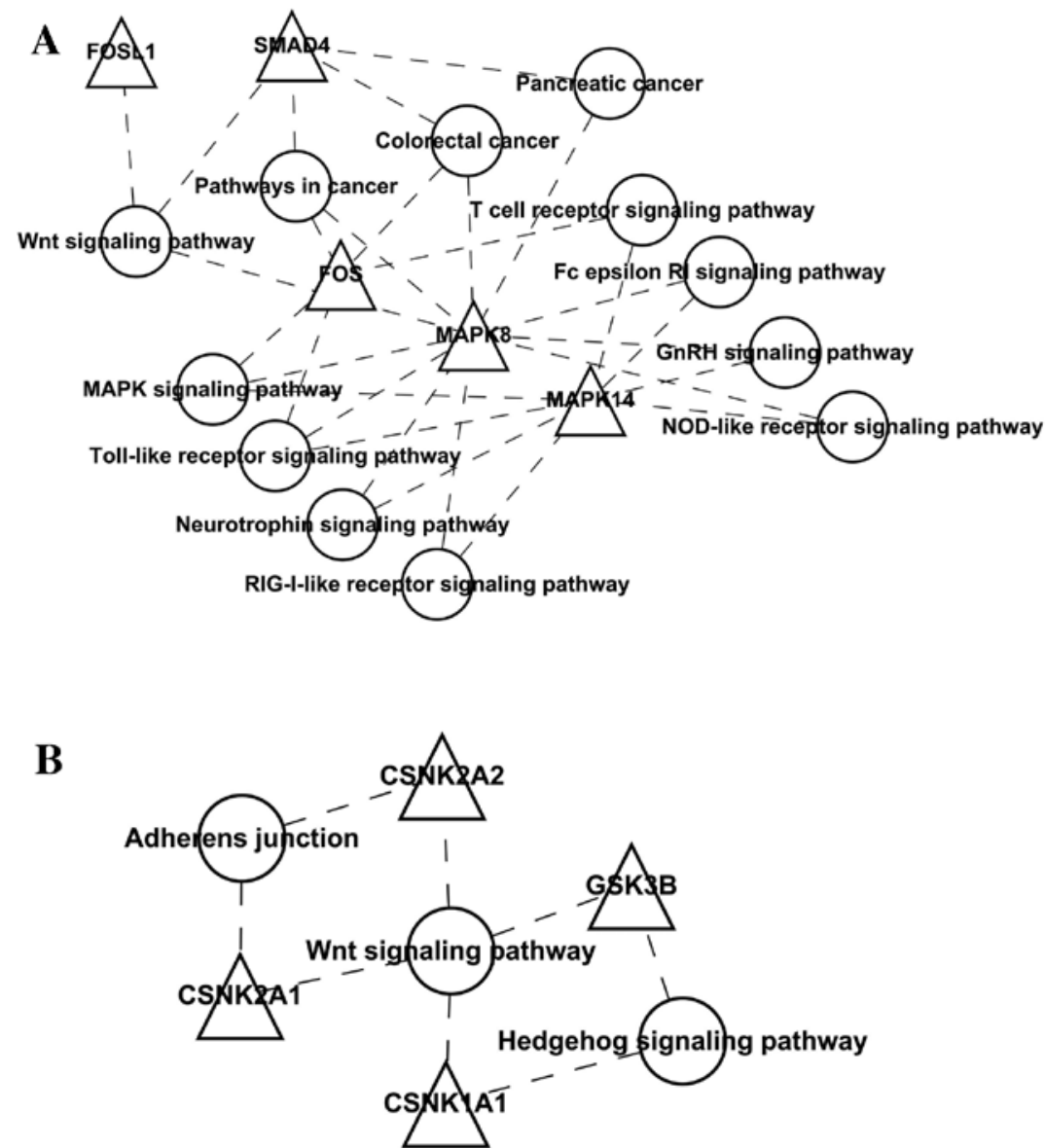

Figure 6. Network of transcription factor regulatory genes and their KEGG function. The ellipse shaped nodes indicate signalling pathways (KEGG); the triangle shaped nodes indicate the regulatory genes of the corresponding transcription factors. (A) The regulatory gene cluster of JUNB; (B) The regulatory gene cluster of EIF2B. KEGG, Kyoto Encyclopedia of Genes and Genomes.

Macrophages are an important element in this complex environment. Monocytes produced by the bone marrow enter the bloodstream and select different tissues for nesting under the effects of corresponding factors. Furthermore, these macrophages display different functions under the effects of the microenvironment after they enter the tissues. For example, alveolar macrophages express lipoxidase at high levels; peritoneal macrophages and hepatic Kupffer cells have relatively potent phagocytotic functions and play antitumor roles (5); and animal tests have shown that knockout of Kupffer cells can improve the metastatic rate of tumors (6). When monocytes migrate to tumor tissues, they differentiate into TAMs in the TME. TAMs may have dual functions in tumor tissues and act as a 'double-edged sword' (7). Macrophages have plasticity and multi-directional differentiation capability (8) and may have different activation types and play different roles. TAMs can be divided into 2 types of macrophages: M1 and M2 types. Both of these 2 types of macrophages can be found in tumors; however, the macrophages in tumors are mainly M1 type in the early stages of tumorigenesis and progression, and these macrophages play roles in immune surveillance and antitumor processes. In contrast, the macrophages in advanced-stage tumors are mainly M2 type, which function in promoting tumor growth, invasion and metastasis.

Previous subjects of investigations have mainly been TAMs and their secreted cytokines. Studies on tumor cells have mainly focused on their growth, metastasis and vascularization, while studies concerning the changes in gene expression and other aspects in tumor cells under the effects of macrophages are scarce. The present study identified 40 differentially expressed genes after microarray data analysis and a comprehensive bioinformatic analysis of these genes.

Through the functional enrichment analysis of the differentially expressed genes, the present study found that among the top 15 GO terms, 9 were related to 'stress' and 'response', indicating that tumor cells were stimulated by various factors that exist in the macrophage-conditioned medium and underwent stress responses. Further investigation is warranted to determine whether these responses are beneficial or detrimental.

The analysis showed that 5 of the differentially expressed genes were related to lung cancer, 2 genes were related to breast cancer, and 1 gene was related to endometrial carcinoma, indicating that these genes have cancer-promoting functions.

After the construction and analysis of a visualization network, we found that 6 differentially expressed genes were related to the 'cell cycle', 4 genes were related to 'cell proliferation', 3 genes were related to 'DNA replication', 7 genes were related to 'intracellular signaling cascades' and 3 genes were related to 'blood vessel morphogenesis'. All of these GO terms were related to tumorigenesis and progression, indicating that the tumor cells underwent changes beneficial for 


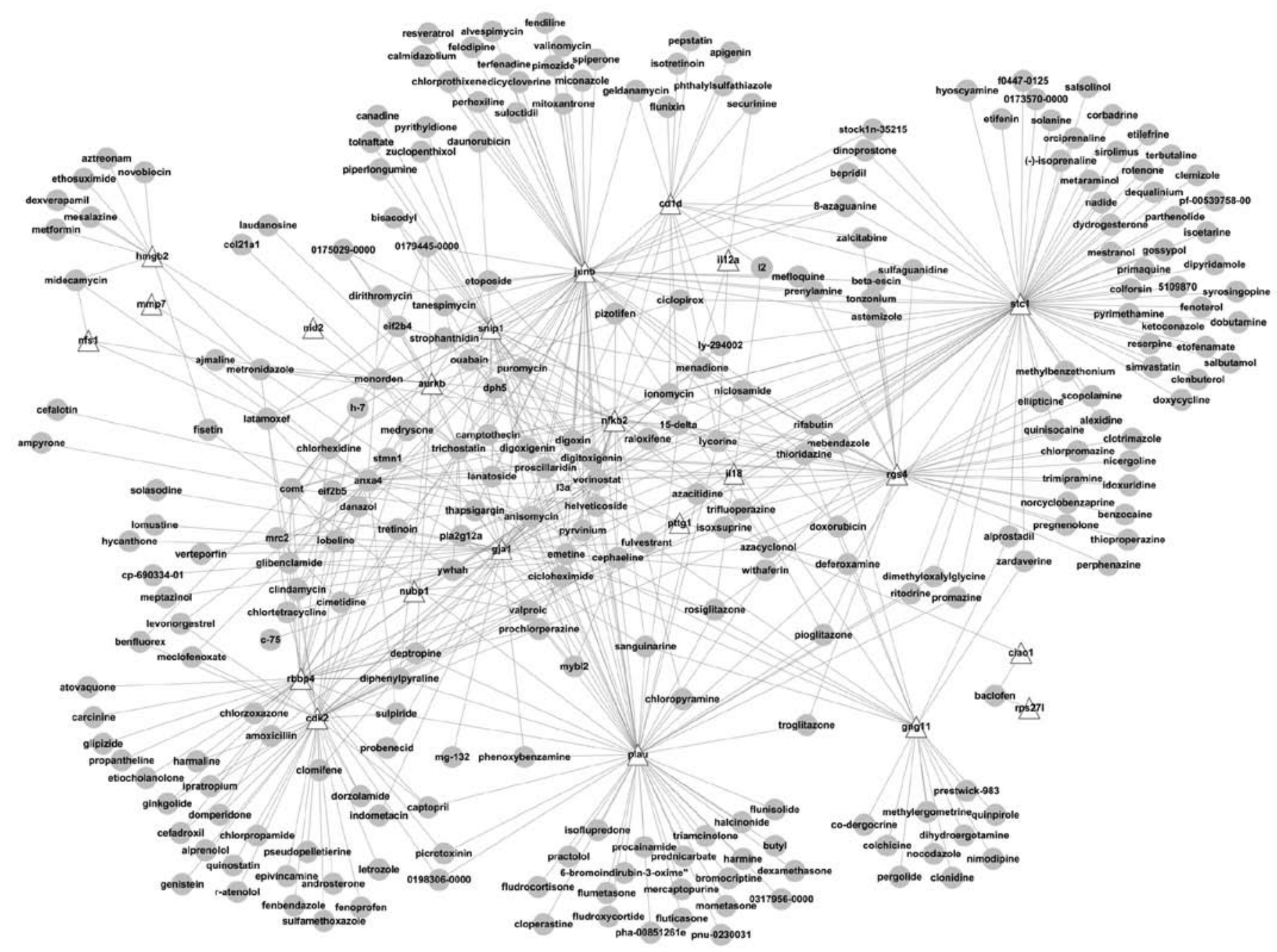

Figure 7. Network of small molecules and their regulated genes. The ellipse-shaped nodes indicate small molecules; the triangle-shaped nodes indicate their regulated genes.

the progression of tumors under the effects of the macrophageconditioned medium.

Many studies have reported on the functions of TAMs in promoting tumor growth, and several studies have shown that TAMs can express several types of cytokines, such as EGF, PDGF, TGF- $\beta 1$, HGF, EGFR and bFGF, that stimulate the proliferation and survival of tumor cells $(9,10)$. It has been confirmed by in vitro co-incubation of tumor cells and macrophages that the substances secreted by macrophages can stimulate the proliferation of tumor cells (11). In vivo tests have shown, through the depletion of macrophages, that TAMs are essential for the growth of different types of tumors (12). GO terms such as 'cell cycle', 'cell proliferation' and 'DNA replication' identified in the present study also support this opinion.

Vascularization is an important event during tumor growth and metastasis. White et al (13) found that the medium for co-incubation of human peripheral blood monocytes and A549 cells can increase the chemotaxis of vascular endothelial cells. Lin et al (14) found in their studies of transgenic Csfop/Csfop animals that TAMs were involved in the metastasis of tumors and were 'the switch for vascularization'. In a PyMT-induced breast cancer model in mice, the knockout of macrophages was found to significantly decrease the pulmonary metastasis of tumors, indicating that TAMs are important for establishing metastatic foci in tumors (15).

It was found that 6 differentially expressed genes were related to the 'extracellular matrix'. In vitro tests have shown that the co-incubation of macrophages and tumor cells can improve the TNF- $\alpha$ and MMP (matrix metalloproteinase)dependent invasive characteristics of tumor cells (16). MMPs are a group of zinc-dependent matrix degrading enzymes, and MMP expression can damage tissue structures and the basilar membrane, which is beneficial for the growth, metastasis and spreading of tumor cells.

The above analysis demonstrated that a number of genes in lung cancer cells under the functions of macrophages underwent changes in their expression levels; consequently the incidence and progression of tumors were promoted. To carry out this analysis, we bioinformatically screened out 5 differentially expressed transcription factors: EIF2B4, EIF2B5, JUNB, GNG11 and HMGB2. Furthermore, the GO annotations for these transcription factors were all related to 'stress' and 'response', indicating that these factors may be important regulatory factors for the changes in tumor cells and they may be potential targets for regulating the growth of tumor cells. 


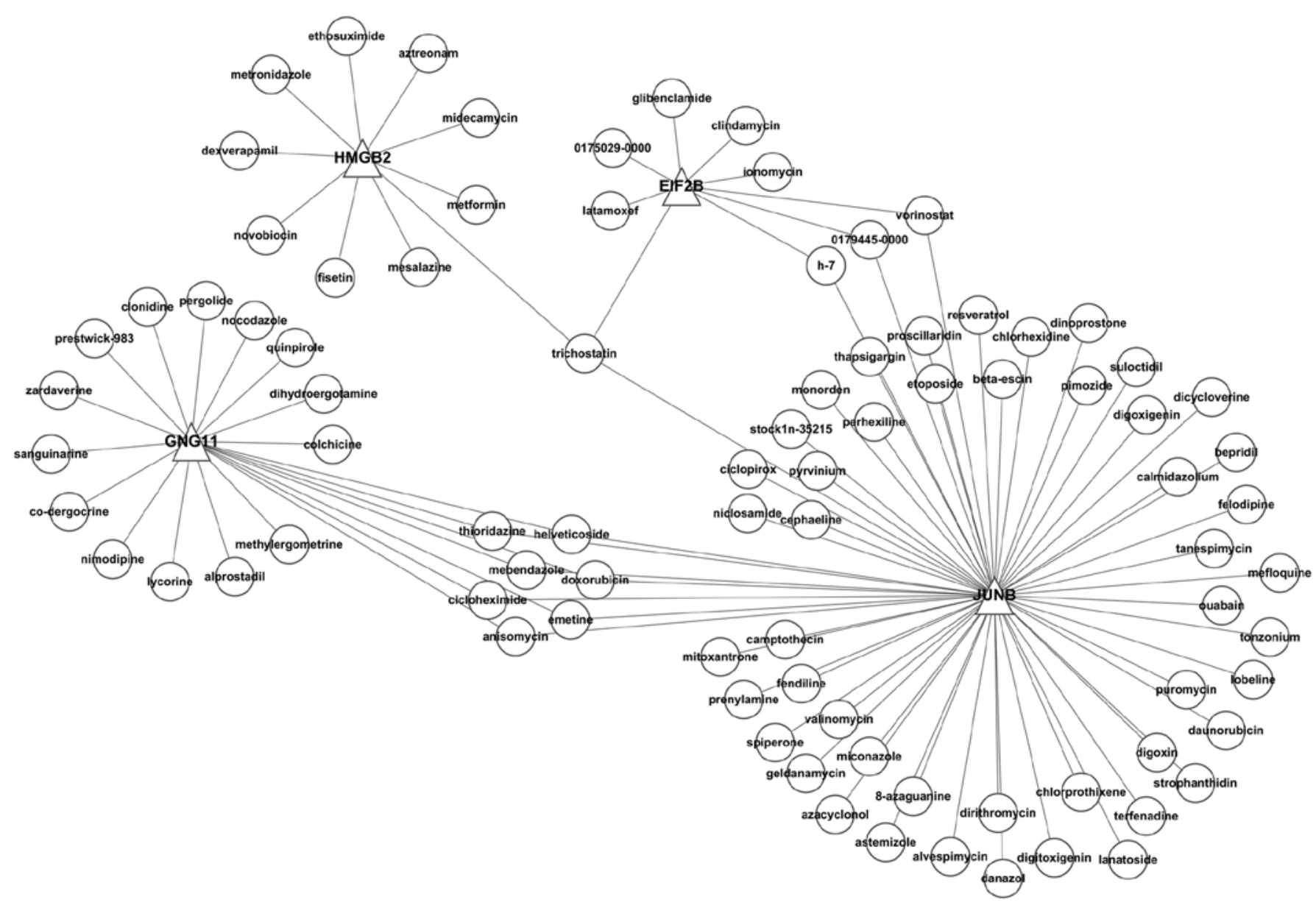

Figure 8. Network of small molecules and their regulated transcription factors. The ellipse-shaped nodes indicate small molecules; the triangle-shaped nodes indicate their regulated transcription factors.

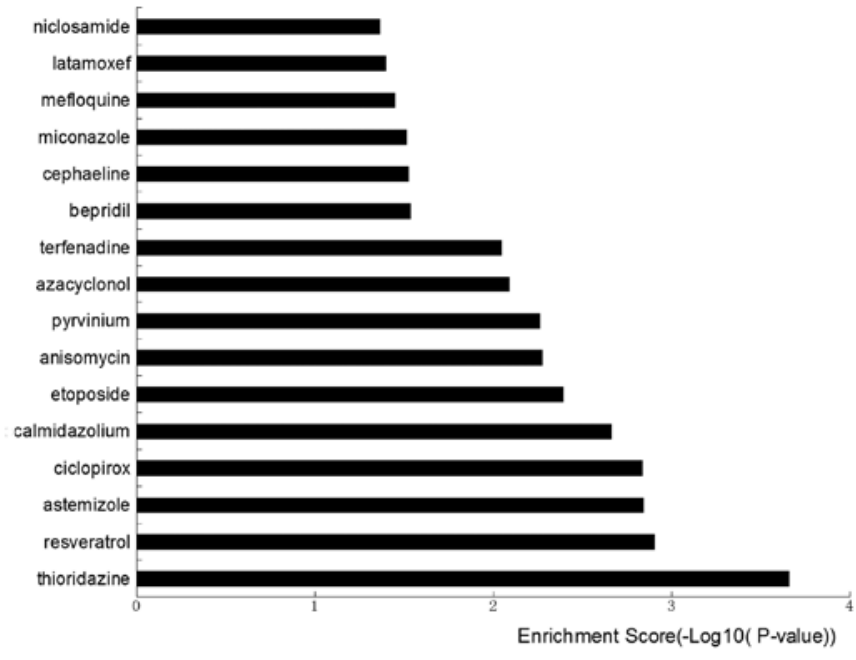

Figure 9. Ranking of the small molecules that may act on transcription factors.

Transcription factors are regulatory factors required for gene expression in all organisms. They are considered to have switching functions and can trigger gene expression by binding to DNA or silencing genes by not binding to DNA. Data mining showed that JUNB and EIF2B were differentially expressed in tumors.
JUNB is a negative growth factor for cell proliferation. In terms of cell cycle regulation, JUNB can promote the growth of osteoblasts and chondroblasts by directly activating the transcription of cyclin A (17). When JUNB is depleted, anoxiainduced VEGF expression is inhibited. Studies in teratomas have also shown that the neovascular rates and degrees are both significantly decreased (18), which indicates that JUNB molecules may be new targets for inhibiting vascularization in tumors. It has been shown that the depletion of JUNB may lead to the upregulation of RAE- $\varepsilon$ on the surface of target cells, which would activate the killing functions of NK cells on target cells (19). This result indicates that the high expression level of JUNB may function in the immunological escape of tumor cells during incidence and tumor progression.

Eukaryotic initiation factors (EIFs) are a group of proteins that play important roles in the initiation of translation in eukaryotic cells. EIF2B is a large complex composed of 5 subunits, and many human diseases have been shown to be directly or indirectly related to EIF2B. In regards to tumors, the functions of EIF2B are related to the GSK-3 protein. Many models for metastasis and the incidence of cancer have shown that the intracellular signaling pathways regulating EIF2B are different from those in normal cells.

To illustrate the functions of these transcription factors, we carried out bioinformatic analysis. Generally, a transcription 
factor can regulate many genes, and a gene may be regulated by many transcription factors, thus creating complex regulatory networks. We constructed a visualization network for transcription factors and their regulatory genes to investigate the functions of the gene cluster regulated by the transcription factor and further illustrate the functions of these transcription factors.

In the KEGG functional enrichment analysis for the regulatory gene assemblies of the transcription factors, the gene assemblies regulated by GNG11 and HMGB2 did not show functional enrichment, while the gene assemblies regulated by EIF2B and JUNB obtained enrichment results.

The 'Wnt signaling pathway' showed enrichments in the two gene assemblies. The 'Wnt signaling pathway' is a wellknown carcinogenic signaling pathway. Wnt protein, encoded by the Wnt gene, can trigger the intracellular signaling transduction pathway, which is involved in cell proliferation, differentiation and migration. It was found that a number of genes in the Wnt/ $\beta$-catenin signaling pathway, such as MMP7, CIM4, VEGF, and E-cadherin, are related to invasion and metastasis (20-22). Previous studies have confirmed that over-activity of Wnt signaling may lead to the hyperplasia of stem cells and malignant transformation into tumor stem cells $(23,24)$. It has been reported that the 'Wnt signaling pathway' can be used in the research and development of antitumor drugs and as a therapeutic target for antitumor therapy. The enrichment of the 'Wnt signaling pathway' in the gene clusters regulated by EIF2B and JUNB indicates that the cells underwent changes beneficial for tumor growth under the functions of these 2 transcription factors.

The 'MAPK signaling pathway' was also enriched among the gene assemblies regulated by JUNB. The 'MAPK signaling pathway' is the common pathway for extracellular signals to induce nuclear reactions (DNA transduction, cell proliferation, apoptosis). The MAPK family is involved in cell growth, development, division, synchronization of intercellular functions and other physiological processes closely related to tumors. The intensive studies on tumors in recent years have shown that the signaling transduction pathway does not function independently, and a large amount of crosstalk can be found among the signaling pathways. Many results have shown that substantial crosstalk can be found during formation and development of tumors between the 'Wnt signaling pathway' and the 'MAPK signaling pathway'. When the 'Wnt signaling pathway' or the 'MAPK signaling pathway' becomes disordered, abnormal embryonic development, defects or even early premature death may occur, and this may lead to loss of control in cell proliferation and differentiation, and further induce tumors (25-27).

The 'Toll-like receptor (TLR) signaling pathway', 'NOD-like receptor (NLR) signaling pathway' and 'RIG-Ilike receptor (RLR) signaling pathway' were also found to be enriched in the JUNB gene cluster. These signaling pathways are all involved in pathways expressing various types of pattern recognition receptors (PRRs). Recently, many studies have confirmed that glioma, breast, prostate, lung, ovarian and esophageal cancer cells are all related to the 'Toll-like receptor signaling pathway' (28-31). These studies used microorganism-derived TLR ligand to indicate that the TLR expressed in the tumor cells had functional activities (32). A recent study revealed that several types of endogenous ligands of TLR exist in the TME (33) and these endogenous ligands affect the progression of tumors through the 'Toll-like receptor signaling pathway'. Ikebe et al (34) found that lipopolysaccharide can promote the invasion and progression of pancreatic cancer through the TLR4 signaling pathway. Other pathways, such as the 'Fc $\varepsilon$ RI signaling pathway' and the 'T cell receptor signaling pathway' also play important roles in the immunological features of tumors (35-37).

The functional enrichment analysis showed that the gene assemblies regulated by JUNB enriched many tumor-related functions, such as 'colorectal cancer', 'pathways in cancer' and 'pancreatic cancer', indicating that JUNB plays important roles during the malignant progression of tumor cells under the stimulation of macrophages. These results suggest that extensive research must be conducted to investigate JUNB as a target for blocking this vicious cycle.

Various genes have been correlated with previously known drugs through biological network technique and network pharmacology. These methods can be utilized to explore non-patent 'targeting' drugs and realize 'new utilization for old drugs', thus saving research funds and significantly shortening the cycles for research and development. For example, König et al (38) carried out an RNA interference screening based on the whole genome. They utilized an integrated systematic method and carried out analysis of gene subsets. The results showed that 10 genes were related to the replication of the influenza virus, and thus it was deduced that vATPase, CAMK2B and other small molecule inhibitors may antagonize virus replication after experimental confirmation. Chen et al (39) identified the inhibitor for the heat shock protein HSP90, NVP-AUY922, using this method, and this inhibitor is currently used to treat cholangiocarcinoma. Wei et al (40) identified the mTOR inhibitor, rapamycin, using this method and it is used for the treatment of acute lymphocytic leukemia. The present study screened thioridazine, resveratrol, astemizole, ciclopirox, calmidazolium, etoposide, anisomycin, pyrvinium, azacyclonol and terfenadine based on their interactions with transcription factors. These interactions may inhibit the progression of pulmonary cancer cells under the effect of macrophage-conditioned medium by inhibiting JUNB and other transcription factors. The results of the present study may provide new clues for further antitumor therapy and research.

\section{Acknowledgements}

This research was supported by the Research Fundation of the Education Department of Heilongjiang, China (no. 12531230).

\section{References}

1. Mantovani A, Bottazzi B, Colotta F, Sozzani S and Ruco L: The origin and function of tumor-associated macrophages. Immunol Today 13: 265-270, 1992

2. Hildenbrand R and Schaaf A: The urokinase-system in tumor tissue stroma of the breast and breast cancer cell invasion. Int $\mathrm{J}$ Oncol 34: 15-23, 2009.

3. Lewis CE and Pollard JW: Distinct role of macrophages in different tumor microenvironments. Cancer Res 66: 605-612, 2006.

4. Gentleman RC, Carey VJ , Bates DM, et al: Bioconductor: open software development for computational biology and bioinformatics. Genome Biol 5: R80, 2004. 
5. Purohit V, Rapaka R, Kwon OS and Song BJ: Roles of alcohol and tobacco exposure in the development of hepatocellular carcinoma. Life Sci 92: 3-9, 2013.

6. Heuff G, Oldenburg HS, Boutkan H, et al: Enhanced tumor growth in the rat liver after selective elimination of Kupffer cells. Cancer Immunol Immunother 37: 125-130, 1993.

7. Chen JJ, Lin YC, Yao PL, et al: Tumor-associated macrophages: the double-edged sword in cancer progression. J Clin Oncol 23: 953-964, 2005.

8. Goetze K, Walenta S, Ksiazkiewicz M, Kunz-Schughart LA and Mueller-Klieser W: Lactate enhances motility of tumor cells and inhibits monocyte migration and cytokine release. Int J Oncol 39: 453-463, 2011.

9. De Palma M and Lewis CE: Macrophage regulation of tumor responses to anticancer therapies. Cancer Cell 23: 277-286, 2013.

10. Horton LW, Yu Y, Zaja-Milatovic S, Strieter RM and Richmond A: Opposing roles of murine duffy antigen receptor for chemokine and murine CXC chemokine receptor-2 receptors in murine melanoma tumor growth. Cancer Res 67: 9791-9799, 2007.

11. Smith HA and Kang Y: The metastasis-promoting roles of tumor-associated immune cells. J Mol Med 91: 411-429, 2013.

12. Sawant A, Hensel JA, Chanda D, et al: Depletion of plasmacytoid dendritic cells inhibits tumor growth and prevents bone metastasis of breast cancer cells. J Immunol 189: 4258-4265, 2012.

13. White ES, Strom SR, Wys NL and Arenberg DA: Non-small cell lung cancer cells induce monocytes to increase expression of angiogenic activity. J Immunol 166: 7549-7555, 2001.

14. Lin EY, Li JF, Gnatovskiy L, et al: Macrophages regulate the angiogenic switch in a mouse model of breast cancer. Cancer Res 66: 11238-11246, 2006.

15. Lin EY, Nguyen AV, Russell RG and Pollard JW: Colonystimulating factor 1 promotes progression of mammary tumors to malignancy. J Exp Med 193: 727-740, 2001.

16. Hagemann T, Robinson SC, Schulz M, et al: Enhanced invasiveness of breast cancer cell lines upon co-cultivation with macrophages is due to TNF- $\alpha$ dependent up-regulation of matrix metalloproteases. Carcinogenesis 25: 1543-1549, 2004.

17. Andrecht S, Kolbus A, Hartenstein B, Angel P and SchorppKistner M: Cell cycle promoting activity of JunB through cyclin A activation. J Biol Chem 277: 35961-35968, 2002.

18. Schmidt D, Textor B, Pein OT, et al: Critical role for NF- $\mathrm{KB}$ induced JunB in VEGF regulation and tumor angiogenesis. EMBO J 26: 710-719, 2007.

19. Nausch N, Florin L, Hartenstein B, et al: Cutting edge: the AP-1 subunit JunB determines NK cell-mediated target cell killing by regulation of the NKG2D-ligand RAE-1ع. J Immunol 176: 7-11, 2006.

20. Hussaini IM, Trotter C, Zhao Y, Abdel-Fattah R, et al: Matrix metalloproteinase-9 is differentially expressed in nonfunctioning invasive and noninvasive pituitary adenomas and increases invasion in human pituitary adenoma cell line. Am J Pathol 170: 356-365, 2007.

21. Pulukuri SM and Rao JS: Matrix metalloproteinase-1 promotes prostate tumor growth and metastasis. Int J Oncol 32: 757-765, 2008.

22. Xu D, McKee CM, Cao Y, et al: Matrix metalloproteinase-9 regulates tumor cell invasion through cleavage of protease nexin-1. Cancer Res 70: 6988-6998, 2010.

23. Kawaguchi-Ihara N, Murohashi I, Nara N and Tohda S: Promotion of the self-renewal capacity of human acute leukemia cells by Wnt3A. Anticancer Res 28: 2701-2704, 2008.
24. Hu J, Dong A, Fernandez-Ruiz V, et al: Blockade of Wnt signaling inhibits angiogenesis and tumor growth in hepatocellular carcinoma. Cancer Res 69: 6951-6959, 2009.

25. Kim D, Rath O, Kolch W and Cho KH: A hidden oncogenic positive feedback loop caused by crosstalk between Wnt and ERK pathways. Oncogene 26: 4571-4579, 2007.

26. Jeong AY, Lee MY, Lee SH, Park JH and Han HJ: PPARס agonist-mediated ROS stimulates mouse embryonic stem cell proliferation through cooperation of p38 MAPK and Wnt/ $\beta$ catenin. Cell Cycle 8: 611-619, 2009.

27. Gazitt Y, Kolaparthi V, Moncada K, Thomas C and Freeman J: Targeted therapy of human osteosarcoma with 17AAG or rapamycin: Characterization of induced apoptosis and inhibition of mTOR and Akt/MAPK/Wnt pathways. Int J Oncol 34: 551-561, 2009.

28. Samara KD, Antoniou KM, Karagiannis K, et al: Expression profiles of Toll-like receptors in non-small cell lung cancer and idiopathic pulmonary fibrosis. Int J Oncol 40: 1397-1404, 2012.

29. Tanaka J, Sugimoto K, Shiraki K, et al: Functional cell surface expression of toll-like receptor 9 promotes cell proliferation and survival in human hepatocellular carcinomas. Int J Oncol 37: 805-814, 2010.

30. Takala H, Kauppila JH, Soini Y, et al: Toll-like receptor 9 is a novel biomarker for esophageal squamous cell dysplasia and squamous cell carcinoma progression. J Innate Immun 3: 631-638, 2011.

31. Qiu J, Shao S, Yang G, Shen Z and Zhang Y: Association of Toll like receptor 9 expression with lymph node metastasis in human breast cancer. Neoplasma 58: 251-255, 2011.

32. Lotze MT,Zeh HJ, Rubartelli A, et al: The grateful dead: damageassociated molecular pattern molecules and reduction/oxidation regulate immunity. Immunol Rev 220: 60-81, 2007.

33. Sato Y, Goto Y, Narita N and Hoon DS: Cancer cells expressing Toll-like receptors and the tumor microenvironment. Cancer Microenviron 2 (Suppl 1): 205-214, 2009.

34. Ikebe M, Kitaura Y, Nakamura M, et al: Lipopolysaccharide (LPS) increases the invasive ability of pancreatic cancer cells through the TLR4/MyD88 signaling pathway. J Surg Oncol 100: 725-731, 2009.

35. Agha-Mohammadi S and Lotze MT: Immunomodulation of cancer: potential use of selectively replicating agents. J Clin Invest 105: 1173-1176, 2000.

36. Tsai JP, Chen HW, Cheng ML, et al: Analysis of host versus tumor interaction in cancer patients: opposing role of transforming growth factor- $\beta 1$ and interleukin- 6 in the development of in situ tumor immunity. Immunobiology 210: 661-671, 2005.

37. Barbieri G, Rimini E and Costa MA: Effects of human leukocyte antigen (HLA)-DR engagement on melanoma cells. Int $\mathrm{J}$ Oncol 38: 1589-1595, 2011.

38. König R, Stertz S, Zhou Y, et al: Human host factors required for influenza virus replication. Nature 463: 813-817, 2010.

39. Chen MH, Lin KJ, Yang WL, et al: Gene expression-based chemical genomics identifies heat-shock protein 90 inhibitors as potential therapeutic drugs in cholangiocarcinoma. Cancer 119: 293-303, 2013.

40. Wei G, Twomey D, Lamb J, et al: Gene expression-based chemical genomics identifies rapamycin as a modulator of MCL1 and glucocorticoid resistance. Cancer Cell 10: 331-342, 2006. 